Mots. Les langages du politique

\title{
Bibliographie de lexicologie socio-politique
}

Josette Lefèvre, Pierre Fiala et Maurice Tournier

\section{(2) OpenEdition}

Journals

Édition électronique

URL : https://journals.openedition.org/mots/5073

DOI : $10.4000 /$ mots. 5073

ISSN : 1960-6001

\section{Éditeur}

ENS Éditions

\section{Édition imprimée}

Date de publication : 1 mars 2004

Pagination : 159-161

ISBN : 2-84788-056-9

ISSN : 0243-6450

\section{Référence électronique}

Josette Lefèvre, Pierre Fiala et Maurice Tournier, «Bibliographie de lexicologie socio-politique », Mots. Les langages du politique [En ligne], 74 | 2004, mis en ligne le 07 mai 2008, consulté le 24 avril 2022. URL : http://journals.openedition.org/mots/5073; DOI : https://doi.org/10.4000/mots.5073 


\section{Bibliographie de lexicologie socio-politique}

Amnesty International, 1998, «Qu'y a-t-il dans un mot? Pour un langage non sexiste des droits humains », Londres-Paris, Amnesty international, $20 \mathrm{p}$.

ANIS J., ESKÉNAZI A., JEANDILLOU J.-F. (textes réunis par), 2002, Le signe et la lettre. Hommage à Michel Arrivé, Paris-Budapest-Turin, L'Harmattan, 482 p.

ARIEL de VIDAS A., 2002, Le tonnerre n'habite plus ici. Culture de la marginalité chez les Indiens teenek (Mexique), Paris, Éditions de l'EHESS, 478 p.

BACOT P., BARATAY E., BARBET D., FAURE O., MAYAUD J.-L. (dir.), 2003, L'animal en politique, Paris, L'Harmattan, $386 \mathrm{p}$.

BAGGIONI D., LARCHER P. (dir.), 1995, Du Sens. Tours, détours et retours du sens dans les sciences humaines d'aujourd'hui, Aix-en-Provence, Publications de l'Université de Provence, $173 \mathrm{p}$.

BERTHOUD A.-C., MONDADA L. (dir.), 2000, Modèles du discours en confrontation, Berne, Peter Lang (Sciences pour la communication), $\mathrm{n}^{\circ} 61,228 \mathrm{p}$.

BOSVELD L., VANDEVELDE D., VAN PETEGHEM M., 2000, De l'indétermination à la qualification. Les indéfinis, Arras, Artois Presses Université, 276 pages.

BOUVIER J.-C., 2003, Espaces du langage. Géolinguistique, toponymie, cultures de l'oral et de l'écrit, Aix-en-Provence, Publications de l'Université de Provence, $414 \mathrm{p}$.

BOYER H., LAGARDE C. (dir.), 2002, L'Espagne et ses langues. Un modèle écolinguistique?, Paris, L'Harmattan, 298 p.

BOYER H., 2003, De l'autre côté du discours. Recherches sur les représentations communautaires, Paris, L'Harmattan, $122 \mathrm{p}$.

BRANCA-ROSOFF, 2001, «La sémantique lexicale du mot «quartier» à l'épreuve du corpus Frantext $\left(19^{\mathrm{e}}-20^{\mathrm{e}}\right.$ siècles $) »$, Langage et société, $\mathrm{n}^{\circ} 96$.

Centrale nationale des employés, 2003, Lexique de la mondialisation. Le syndicalisme dans la mondialisation, Bruxelles, Éditions CNE, 165 p.

CHABIN M.-A. (dir.), 2001, «L'archivage», Document numérique, $\mathrm{n}^{\circ} 4$, Paris, Hermès, $192 \mathrm{p}$.

CHATEAURAYNAUD F., 2003, Prospero, une technologie littéraire pour les sciences humaines, Paris, CNRS (Communication), 406 p.

CHIANTARETTO J.-F., ROBIN R., 2003, «Témoignage et écriture de l'histoire. » Décade de Cerisy, 21-31 juillet 2001, Paris, L'Harmattan, 482 p.

COLLETTA J.-M., NUCHÈZE de V., 2002, Guide terminologique pour l'analyse des discours. Lexique des approches pragmatiques du langage, Berne, Peter Lang (Sciences pour la communication), $222 \mathrm{p}$. 
CORDIER F., FRANÇOIS J. (dir.), 2002, Catégorisation et langage, Paris, Hermès, $232 \mathrm{p}$.

CORTEN A., 1999, Alchimie politique du miracle. Discours de la guérison divine et langue politique en Amérique latine, Montréal, Éditions Balzac, 252 p.

CORTEZ Y., 2002, Le Français que l'on parle. Son vocabulaire, sa grammaire, ses origines, Paris, L'Harmattan, 210 p.

COTTEREAU A., LADRIĖRE P. (éd.), 1992, Pouvoir et légitimité. Figures de l'espace public, Raisons pratiques, 3, Paris, Éditions EHESS, 296 p.

DAILLE B., ROMARY L. (dir.), 2002, «Linguistique de corpus », Traitement automatique des langues, $\mathrm{n}^{\circ}$ 42, Paris, Hermès, $310 \mathrm{p}$.

DENDALE P., TASMOWSKI L. (dir.), 2001, Le conditionnel en français, Metz, Université de Metz, Recherches linguistiques.

DESSALLES J.-L., 2000, Aux origines du langage. Une histoire naturelle de la parole, Paris, Hermès, $352 \mathrm{p}$.

DIDRY C., 2002, Naissance de la convention collective. Débats juridiques et luttes sociales en France au début du $20^{e}$ siècle, Paris, Éditions de l'EHESS, 268 p.

DONEUX J.-L., 2003, Histoire de la linguistique africaine, Aix-en-Provence, Publications de l'Université de Provence, $266 \mathrm{p}$.

DURAND G., LOGOSSAH K., Les noms de famille d'origine africaine de la population martiniquaise d'ascendance servile, Paris, L'Harmattan, $354 \mathrm{p}$.

ÉLOY J.-M., BLOT D., CARCASSONNE M., LANDRECIES J. (dir.), 2003, Français, Picard, immigrations. Une enquête épilinguistique, Paris, L'Harmattan, 285 p.

FÉNOGLIO I. (dir.), 2003, «Écritures en acte et genèse du texte», Langage et Société, $\mathrm{n}^{\circ} 103$, mars, 166 pages.

GARDES TAMINE J. (dir.), 2003, L'Allégorie corps et âme, Aix-en-Provence, Publications de l'Université de Provence, $252 \mathrm{p}$.

GIACOMI A., STOFFEL H., VÉRONIQUE D. (dir.), 2000, Appropriation du français par des marocains arabophones à Marseille: bilan d'une recherche, Aix-enProvence, Publications de l'Université de Provence, 344 p.

GRAMACCIA G., 2001, Les actes de langage dans les organisations, Paris, L'Harmattan, $288 \mathrm{p}$.

JULIA C., 2001, Fixer le sens? La sémantique spontanée des gloses de spécification $d u$ sens, Paris, Presses de la Sorbonne-Nouvelle, 300 p.

KHAZNADAR E., 2002, Le féminin à la française, Paris, L'Harmattan, 239 p.

LAVER M., BENOIT K., GARRY J., 2003, «Extracting Policy Positions from Political Texts Using Words as Data », American Political Science Review, n 7/2, p. 311-331.

LAVOINNE Y. 2003, L’humanitaire et les médias, Lyon, Presses Universitaires de Lyon, $138 \mathrm{p}$.

LEE N.-S., 2003, Identité langagière du genre. Analyse du discours éditorial, Paris, L'Harmattan, $220 \mathrm{p}$.

MARCELLESI J.-B., et autres., 2003, Sociolinguistique, épistémologie, langues régionales, polynomie, Paris, L'Harmattan, $306 \mathrm{p}$.

MARTIN R., 2002, Comprendre la linguistique, Paris, PUF (Quadrige), 190 p.

MELANDRI P., 1997, «La rhétorique populiste aux États-Unis», Vingtième siècle, $\mathrm{n}^{\circ} 56$. 
MICHARD C., 2002, Sexe en linguistique. Sémantique ou zoologie?, Paris, L'Harmattan, $160 \mathrm{p}$.

MINEL J.-L., 2002, Filtrage sémantique. Du résumé automatique à la fouille de textes, Paris, Hermès, $202 \mathrm{p}$.

MOUCHTOURIS A., 2003, Les jeunes de la nuit. Représentations sociales des conduites nocturnes, Paris, L'Harmattan, $126 \mathrm{p}$.

MOUSSA S. (dir.), 2003, L'idée de «race» dans les sciences humaines et la littérature (18 et $19^{e}$ siècles), Paris, L'Harmattan, $472 \mathrm{p}$.

NICOLAS J., 2002, La rébellion française. Mouvements populaires et conscience sociale (1661-1789), Paris, Seuil, 609 p.

PAVEAU M.-A., SARFATI G.-É., 2003, Les grandes théories de la linguistique. De la grammaire comparée à la pragmatique, Paris, Armand Colin, $256 \mathrm{p}$.

QUATREVAUX A., 2002, Journaux de formation, analyse de discours et communication orale, Paris, L'Harmattan, $234 \mathrm{p}$.

RÉMY-GIRAUD S., PANIER L. (dir.), 2003, La polysémie ou l'empire des sens, Lyon, Presses Universitaires de Lyon, $320 \mathrm{p}$.

RIVIÈRE D'ARC H., 2001, Nommer les nouveaux territoires urbains, Paris, MSH Éditions et Éditions UNESCO, 280 p.

ROULET E., FILLIETTAZ L., GROBET A., 2001, Un modèle et un instrument d'analyse de l'organisation du discours, Berne, Peter Lang (Sciences pour la communication), $\mathrm{n}^{\circ} 62,405 \mathrm{p}$.

SEGOND F, (dir.), 2002, Multilinguisme et traitement de l'information. Traité des sciences et techniques de l'information, Paris, Hermès, $256 \mathrm{p}$.

STEUCKARDT A., NIKLAS-SALMINEN A. (dir.), 2003, «Le mot et sa glose», Langues et langage, $\mathrm{n}^{\circ}$ 9, Aix-en-Provence, Publications de l'Université de Provence, $308 \mathrm{p}$.

TOURATIER C., 2003, Morphologie et morphématique, Aix-en-Provence, Publications de l'Université de Provence, $324 \mathrm{p}$.

VIARD J. (dir.), 1996, Aux sources du populisme nationaliste, La Tour-d'Aigues, Éditions de l'Aube, $164 \mathrm{p}$.

WEBER F., 2001, Le travail à-côté. Étude d'ethnographie ouvrière, Paris, Éditions de l'EHESS, $216 \mathrm{p}$. 